\title{
OTIOTOMTCS
}

Revista de economía, empresa y sociedad

DIECINUEVE AÑOS DE INVESTIGACIÓN

\section{Las personas y la cadena de suministro}

\section{Milena Gómez-Gedeño}

Profesora de Ingeniería Industrial. Universidad Tecnológica de Panamá

\section{Laura Guitart-Tarrés}

Profesora de Economía y Empresa (UB). Profesora colaboradora de los Estudios de Economía y Empresa (UOC)

\section{Shantall Morantes Guerra}

Universidad Tecnológica de Panamá. Facultad de Ingeniería Industrial

\section{Yohana Li Zeng}

Universidad Tecnológica de Panamá. Facultad de Ingeniería Industrial

RESUMEN La gestión de los recursos humanos (HRM) con un enfoque hacia la cadena de suministro (SC) permite a las empresas gestionar eficazmente sus cadenas de suministros. Este artículo demuestra la importancia del estudio de los recursos humanos en la cadena de suministro (HRSC) realizando un análisis en profundidad de las investigaciones en estos dos campos (HR y SC) que permite identificar sus potencialidades y sus carencias. En este sentido, se han identificado las áreas temáticas abordadas en la literatura y sus principales contribuciones, así como la existencia de lagunas en la literatura. A partir del análisis efectuado, se han localizado 53 publicaciones que destacan el potencial de la HRSC. Los resultados muestran que en los últimos cuatro años, entre 2012 y 2017, se ha dado un incremento significativo del $49 \%$ de las investigaciones sobre HRSC.

PALABRAS CLAVE cadena de suministro (SC); recurso humano (HR); gestión de la cadena de suministro (SCM); gestión de los recursos humanos (HRM) 


\title{
People in the Supply Chain: 19 years of research
}

\begin{abstract}
Human Resources Management (HRM) with a focus on Supply Chain Management (SCM) empowers companies to effectively manage their supply chains. This article justifies the importance of the study of Human Resources in the Supply Chain (HRSC) and provides an in-depth analysis of research in these two fields, which reveals their potentialities and shortcomings. Thus, the thematic areas addressed have been identified, as well as their main contributions and the existence of gaps in the literature. From the analysis, 53 publications have been identified that highlight the potential of HRSC. The results show that, in the last four (4) years between 2012 and 2017, there has been a significant increase of 49\% in HRSC research.
\end{abstract}

KEYWORDS Supply Chain (SC); Human Resources (HR); Supply Chain Management (SCM); Human Resources Management (HRM)

\section{Introducción}

La gestión de la cadena de suministro (SCM) se basa en la integración de los procesos clave de negocio en el suministro de productos, servicios e información, que añaden valor, desde los proveedores hasta el usuario final. En este sentido, según Gattorna (2006), se considera generalmente que la cadena de suministro es una mezcla 50/50 entre infraestructura y tecnología (aspectos hard o estructurales) cuando más bien es 45/45/10, como mezcla del comportamiento humano (soft), la tecnología de la información y la infraestructura.

El estudio desarrollado por Fawcett et al. (2008), sobre los diez beneficios, barreras y puentes para lograr el éxito de la SCM ${ }^{1}$, concluye que los principales obstáculos se encuentran en los aspectos estructurales o fuertes (tecnología, información y sistemas de medición, entre otros), mientras que los problemas relacionados con las personas (como la ausencia de confianza, la aversión al cambio, la falta de compromiso, culturas incompatibles y las malas prácticas de HR) son más difíciles de solventar. Sin embargo, las personas podrían ser la clave para el éxito de la SCM y deben ser tomadas en cuenta con el mismo énfasis con el que son atendidas las principales barreras.

1. A través de un análisis cuantitativo y cualitativo, mediante encuestas por correo y el análisis de 51 casos en profundidad. 
Giunipero et al. (2008)2 analizan las investigaciones publicadas en un período de diez años (desde 1996 a 2006) sobre cadena de suministro (SC), evidenciando que algunas categorías son ampliamente estudiadas, mientras que existen ausencias en otras temáticas. Este es el caso de los recursos humanos (HR), ámbito que tan solo ha sido estudiado en un $2 \%$ de las investigaciones sobre SC.

En conclusión, a pesar de que las personas podrían ser la clave para el éxito de la SCM, los recursos humanos (HR) son casi inexistentes en las investigaciones sobre SC. En esta línea, con la intención de visibilizar las potencialidades y las carencias existentes en el estudio de los recursos humanos en la cadena de suministro (HRSC), este trabajo se plantea las siguientes preguntas: 1) ¿qué áreas temáticas han sido analizadas en las investigaciones sobre SCM?; 2) ¿existen lagunas dentro de la literatura?; 3) ¿está el ámbito de las personas suficientemente analizado dada su importancia en la SC?; 4) ¿hacia dónde deberían dirigirse las investigaciones futuras?

Este artículo presenta, en primer lugar, un análisis de las publicaciones existentes. En segundo lugar, se realiza un análisis bibliométrico (desde 1999 a 2017) sobre la HRSC (frecuencia de publicación, metodologías utilizadas y clasificación por categorías temáticas) para, por último, establecer las más relevantes sobre HRSC.

\section{Publicaciones sobre recursos humanos y cadena de suministros}

En la literatura académica existen dos revisiones bibliográficas sobre la gestión de las personas en la cadena de suministro: Gómez-Cedeño et al. (2014)³ y Hohenstein et al. (2014)4. Ambos trabajos destacan la escasez de investigaciones sobre recursos humanos y cadena de suministros.

En el estudio de Gómez-Cedeño et al. (2014), se localiza un total de 46 artículos sobre HRSC, en 29 revistas científicas de diversas disciplinas. Además, se efectúa una clasificación de los artículos identificados, agrupándolos en cuatro temas: 1) competencias/perfiles de los directivos y personal de apoyo de SCM

2. Este autor analiza las investigaciones realizadas sobre SC a través de una revisión de la literatura basada en la clasificación de temas que propone el Institute for Supply Chain Management (ISM).

3. Estos autores realizan un estudio bibliométrico para conocer la escasez de investigaciones existentes sobre HRSC (1997 a 2012).

4. Se trata de una revisión de la literatura que analiza los problemas de HRSC en las publicaciones durante dos décadas (1998-2014). 
(35 \%); 2) impacto y mejora de la SCM a través de la HRM (35 \%); 3) relaciones con los miembros de la cadena y los HR (17\%); 4) reclutamiento y selección de los directivos y personal de apoyo de SCM (13\%).

Por otro lado, Hohenstein et al. (2014) analizan los problemas de HRSC establecidos en las publicaciones de las doce principales revistas de SCM. Así, identifican 109 investigaciones, categorizadas en siete ámbitos temáticos: 1) habilidades, conocimientos y capacidades; 2) formación y desarrollo; 3) impacto de la HRM en el desempeño; 4) educación y enseñanza; 5) contratación y reclutamiento; 6) compensación y retribución; y 7) mentalidad global en la "cadena de suministro» o en la «gestión de la cadena de suministro». Los resultados muestran que la mayoría de los artículos se focalizan en tres áreas de investigación: 1) habilidades, conocimiento y destreza (95 de 109); 2) formación y desarrollo (85 de 109); y 3) impacto de la HRM en el desempeño (58 de 109). Además, en los últimos años se observa un aumento significativo de las publicaciones sobre habilidades, conocimientos y capacidades, lo cual denota la preocupación de los investigadores sobre la importancia estratégica de encontrar las personas adecuadas que puedan satisfacer los requerimientos de la cadena de suministro.

\section{Análisis bibliométrico (1999-2017): diecinueve años de investigación sobre recursos humanos en la cadena de suministro}

Con el objetivo de conocer la evolución de las investigaciones sobre recursos humanos en la cadena de suministro (HRSC) de los últimos años, se realizó un análisis bibliométrico para el período de diecinueve años comprendido entre los años 1999 a 2018. Se efectuó una búsqueda sistemática, a través de cinco bases de datos científicas: 1) ProQuest, 2) Emerald Insight, 3) Science Direct, 4) Taylor \& Francis y 5) Wiley Online Library. A partir de las palabras clave y secuencias de palabras contenidas en el título, abstract y conclusiones: 1) gestión de la cadena de suministro y/o cadena de suministro; y 2) gestión de recursos humanos, recursos humanos, entre otros. A partir de los resultados se identificaron un total de 53 artículos estrictamente relacionados con los dos ámbitos (HR y SC). Estos fueron localizados en 32 revistas de diversas disciplinas, clasificadas en cuatro áreas temáticas: 1) proceso de capacitación y planificación del personal de apoyo, 2) relaciones entre los miembros de la (SC) y el recurso humano $(\mathrm{HR}), 3)$ impacto del recurso humano en la SC, y 4) habilidades, competencias y capacidades de los miembros de la SC.

La clasificación en cuatro áreas temáticas se realizó para establecer la más analizada en HRSC. En este sentido, se agruparon los artículos según su conte- 
nido (gráfico 1) y se realizó la lectura detallada y en profundidad de los artículos identificados. Los resultados fueron que un $23 \%$ de las publicaciones pertenecían al primer grupo temático 1) Proceso de capacitación y planificación del personal de apoyo; un 23 \% estaban en el ámbito 2) Relaciones entre los miembros de la cadena de suministro (SC) y el recurso humano (HR), un $17 \%$ pertenecía a la categoría (3) Impacto del recurso humano en la cadena de suministro, y finamente, un 38 \% se clasificó en el grupo (4) Habilidades, competencias y capacidades de los miembros de la SC. Por tanto, esta última área temática fue la más estudiada de las cuatro.

Gráfico 1. Clasificación temática de las 53 publicaciones sobre HRSC

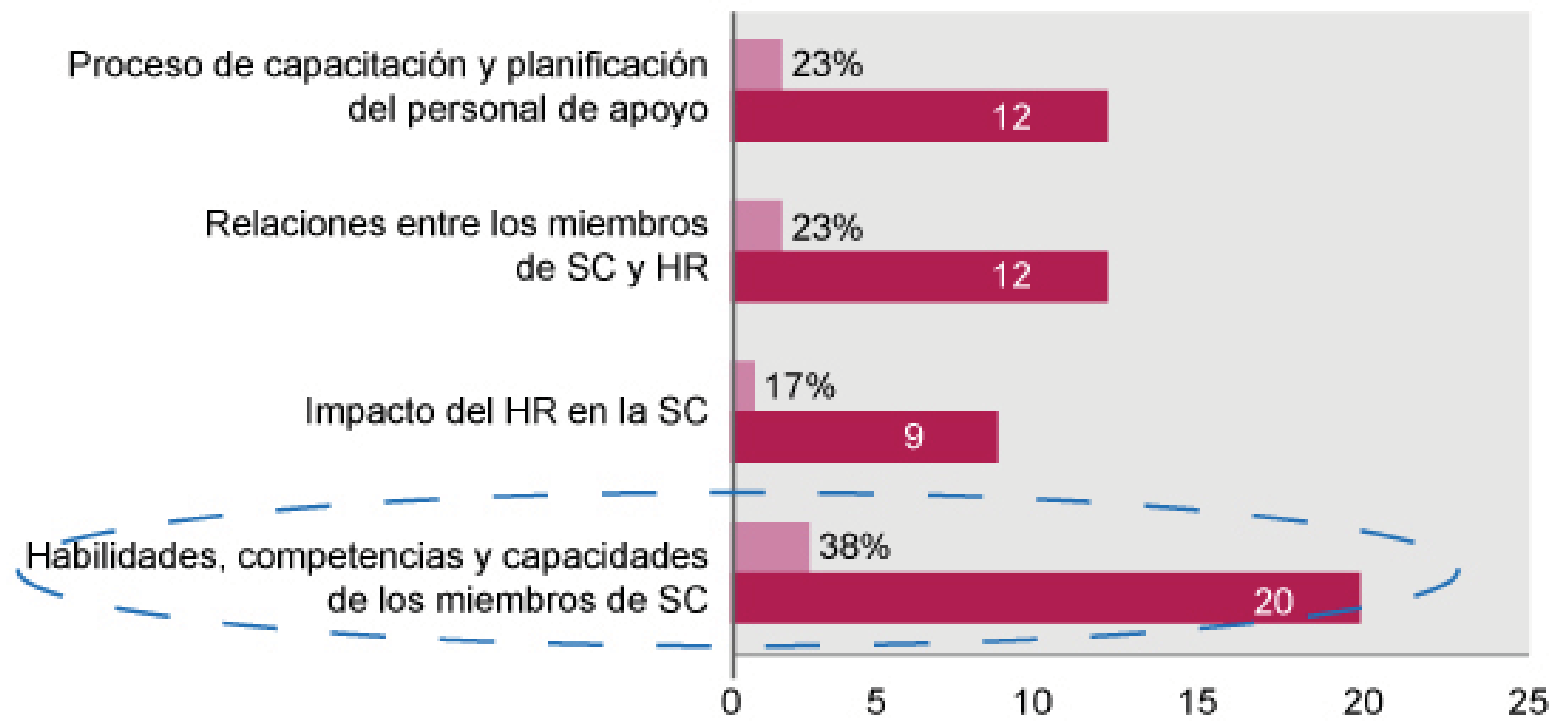

Fuente: elaboración propia.

Por otro lado, se efectuó un análisis de la frecuencia de las publicaciones sobre HRSC, por año de publicación, durante el período comprendido entre los años 1999 a 2017. A pesar de la existencia de grandes vacíos sobre HRSC dentro de la literatura, múltiples investigaciones previas ya destacaron la potencialidad del estudio de HRSC (Gómez-Cedeño et al., 2014; Hohenstein et al., 2014). En esta línea, los resultados muestran un aumento significativo de las publicaciones sobre SCM en los cuatro últimos años, entre el año 2012 al 2017, con veintiséis nuevas publicaciones, lo cual representa el $49 \%$ de las investigaciones sobre HRSC (gráfico 2). 
Gráfico 2. Frecuencia de publicación sobre HRSC, por año

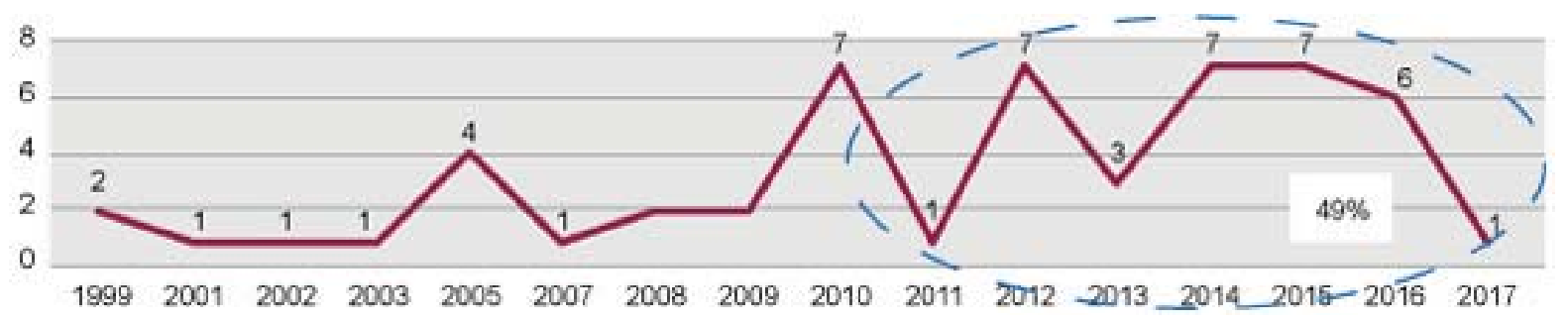

Fuente: elaboración propia.

Se realizó también una clasificación de las publicaciones según el tipo de metodología de investigación utilizada, identificando las que utilizaron métodos empíricos frente a no empíricos, distinción también utilizada por Giunipero et al. (2008). Los resultados indican que la encuesta es la metodología de investigación mayoritariamente utilizada por los investigadores en un $45 \%$ de los artículos. El $34 \%$ de las publicaciones son trabajos teóricos, y el $21 \%$ se basa en el estudio de casos. Estos valores demuestran el creciente interés de los investigadores en los últimos años por medir los factores relacionados con la gestión de la cadena de suministro y los recursos humanos.

\section{Principales contribuciones de las investigaciones sobre recursos humanos en la cadena de suministro}

A partir del análisis del contenido de los 53 artículos sobre recursos humanos en la cadena de suministro (HRSC) identificados en el análisis bibliométrico anterior (1999-2017), la tabla 1 presenta las principales contribuciones por áreas temáticas.

Tabla 1. Principales contribuciones de las publicaciones, por área temática

\begin{tabular}{l|l|}
\hline $\begin{array}{l}\text { Proceso de } \\
\text { capacitación y } \\
\text { planificación del } \\
\text { personal de apoyo }\end{array}$ & $\begin{array}{l}\text { Las prácticas convencionales de SCM diseñadas para } \\
\text { cuadas para fomentar las competencias en logística y en la } \\
\text { SC (Ding et al., 2015). }\end{array}$ \\
\hline $\begin{array}{l}\text { Los incentivos de los empleados están positivamente rela- } \\
\text { cionados con la integración interna y negativamente relacio- } \\
\text { nados con la integración de los clientes (Huo et al., 2015). }\end{array}$
\end{tabular}




\section{Relaciones entre los miembros de la SC y el HR}

\section{Impacto del HRSC}

Las relaciones laborales dentro de la SC están influenciadas por la actitud del empleado hacia el trabajo. Por otro lado, las relaciones laborales dentro de la cadena de suministro pueden optimizarse definiendo y evaluando las competencias laborales (Firescu, 2014).

La flexibilidad de la empresa tiene relación entre el capital humano de la empresa y sus ventajas competitivas, al igual que la conexión entre la flexibilidad de los proveedores y las ventajas competitivas de la empresa (Jin et al., 2010).

El rol emergente del administrador de la SCM hacia gerente estratégico de las relaciones de la cadena de suministro (SCM) pasa de gestionar procesos a gestionar relaciones con proveedores (Wilson y Barbat, 2015).

La integración interna (INTI) de la cadena de suministro tiene un efecto directo en el logro de la satisfacción del cliente, alta flexibilidad y mejor calidad (Alfalla-Luque et al., 2015).

La práctica y la investigación en gestión de recursos humanos se han desarrollado casi exclusivamente dentro de empresas individuales (Fisher et al., 2010).

Tanto las habilidades múltiples de los gerentes como de los empleados tienen una relación positiva con la integración interna de la cadena de suministro (Huo et al., 2016).

Las habilidades percibidas como las más importantes por los profesionales se centran en aquellas actividades que son esenciales para ese rol: liderazgo, resolución de problemas y SRM, entre otras (Wu et al., 2017).

Las necesidades clave de los graduados son: la gestión del tiempo, el aprendizaje colaborativo, el trabajo en equipo y la resolución de problemas (Jordan et al., 2016).

La gestión del talento en la cadena de suministro es una fuente de ventaja competitiva para las empresas en crecimiento (Dubey et al., 2015).

Fuente: elaboración propia.

A partir de las aportaciones de los investigadores que recoge la tabla 1, se pueden sugerir algunas recomendaciones en relación con la gestión de la cadena de suministro. En este sentido, la gestión de recursos humanos debería ampliar su óptica tradicional para albergar también las prácticas necesarias para gestionar correctamente la cadena de suministro en su sentido amplio. El nuevo enfoque debe acoger no solo las competencias laborales necesarias para las relaciones internas en 
la empresa, sino también para la gestión de relaciones con clientes y proveedores. Wilson y Barbat (2015) ya inciden en la figura del gerente estratégico que pase de gestionar procesos a gestionar relaciones con proveedores.

Otra de las sugerencias interesantes que afloran de la literatura es la importancia de la gestión de los recursos humanos y las nuevas habilidades que deben poseer los profesionales. En este sentido, cabe destacar que, tal como establecen Dubey et al. (2015), la gestión del talento en la cadena de suministro es una fuente de ventaja competitiva para las empresas en crecimiento.

\section{Conclusiones}

A pesar de la evolución que ha tenido el campo de estudio de la SC en los últimos años, sigue existiendo una gran confusión en su definición a causa de la amplitud y complejidad del concepto, quedando todavía grandes lagunas en la investigación. El análisis realizado en este trabajo es un buen marco de referencia en el estudio de la cadena de suministro que evidencia un abanico de retos y oportunidades en el planteamiento de futuras investigaciones. El estudio de la HRM en la SC constituye una de estas nuevas oportunidades para la investigación.

Además, los resultados muestran que el número de publicaciones sobre recursos humanos en la literatura de SCM ha aumentado significativamente en los últimos cuatro años (entre 2012 y 2017). Se han identificado 26 nuevas publicaciones, lo cual representa el 49 \% de las investigaciones, tendencia que se prevé que continúe al alza. Ello demuestra el creciente interés de los investigadores hacia los estudios de HRSC.

Conviene destacar también que los resultados obtenidos son coherentes con la literatura previa, ya que investigaciones como las de Gómez et al. (2014) o Hohenstein et al. (2014) ya demostraron que el interés de los investigadores por la HRSC nació hace diecinueve años, aunque aún queda camino por recorrer en la investigación en este ámbito. Esto supone un campo por explorar, lleno de grandes retos y oportunidades. En este sentido, la revisión sistemática realizada en este trabajo contribuye a una mejor comprensión de la gestión de los recursos humanos en la cadena de suministro, así como a valorar su creciente importancia en la literatura de SCM.

Para concluir, Beth et al. (2003) consideran que, a pesar de años de avances de procesos y soluciones tecnológicas, una adaptación ágil de la cadena de suministro sigue siendo un objetivo difícil de alcanzar, y quizá sean las personas quienes dificultan el funcionamiento de la SC. Muchas empresas se han centrado en mejoras e inversiones tecnológicas y de infraestructura, pero el siguiente paso debería ser enfocarse en las personas que gestionan y operan la SC. Este es el reto para alcanzar el éxito de la SC: la gestión de las personas en la cadena de suministros. 


\section{Referencias bibliográficas}

ALFALLA-LUQUE, R.; MARÍN-GARCÍA, J. A.; MEDINA-LÓPEZ, C. (2015). «An analysis of the direct and mediated effects of employee commitment and supply chain integration on organisational performance». International Journal of Production Economics. N. ${ }^{\circ} 162$, págs. 242-257.

BETH, S.; BURT, D. N.; COPACINO, W.; GOPAL, CH.; LEE, H. L.; LYNCH, R. P.; MORRIS, S. (2003). «Supply Chain Challenges: Building Relationships». Harvard Business Review. Vol. 81, n. 7 , págs. 64.

DING, M. J.; KAM, B. H.; ZHANG, J. Y.; JIE, F. (2015). «Effects of human resource management practices on logistics and supply chain competencies-evidence from China logistics service market». International Journal of Production Research. Vol. 53, n. ${ }^{\circ}$ 10, págs. 2.885-2.903.

DUBEY, R.; GUNASEKARAN, A. (2015). «Supply chain talent: the missing link in supply chain strategy». Industrial and Commercial Training. Vol. 47, n. ${ }^{\circ}$, págs. 257-264.

FAWCETT, S.; MAGNAN, M.; MCCARTER, M. (2008). «Benefits barriers and bridges to effective supply chain management». Supply Chain Management: An International Journal. Vol. 13, n. ${ }^{1}$, págs. 35-48.

FIRESCU, V. (2014). «Research Regarding the Optimization of Work Relationships within Supply Chain by Defining and Evaluating Job Competencies». In Applied Mechanics and Materials. Vol. 555, págs. 805-810. Trans Tech Publications.

FISHER, S.; GRAHAM, M.; VACHON, S.; VEREECKE, A. (2010). "Guest Editors' Note: Don't miss the boat: Research on HRM and supply chains». Human Resource Management. Vol. 49, n. ${ }^{\circ} 5$, págs. 813-828.

GATTORNA, J. (2006). Living Supply Chains; How to Mobilize the Enterprise Around Delivering What Your Customers Want. Londres: Financial Times Prentice Hall. GIUNIPERO, L.; HANDFIELD, R.; ELTANTAWY, R. (2006). «Supply management evolution key skill sets for the supply manager of the future». International Journal of Operations \& Production Management. Vol. 26, n. ${ }^{\circ} 7$, págs. 822-844.

GIUNIPERO, L.; HOOKER, R.; MATTHEWS, S.; YOON, T.; BRUDVIG, S. (2008). «A Decade of SCM Literature: Past, Present and Future Implications». Journal of Supply Chain Management. Vol. 44, n. ${ }^{\circ} 4$, págs. 66-86.

GÓMEZ-CEDEÑO, M.; CASTÁN, J. M.; GUITART-TARRÉS, L. (2014). «La importancia de los recursos humanos en la cadena de suministro». Dirección y Organización. N. ${ }^{\circ}$ 54, págs. 13-25.

GÓMEZ-CEDEÑO, M.; CASTÁN-FARRERO, J.; GUITART-TARRÉS, L.; MATUTEVALLEJO, J. (2015). «Impact of human resources on supply chain management and performance». Industrial Management \& Data Systems. Vol. 115, n. ${ }^{\circ}$, págs. 129-157. 
HOHENSTEIN, N. O.; FEISEL, E.; HARTMANN, E. (2014). «Human resource management issues in supply chain management research: a systematic literature review from 1998 to 2014». International Journal of Physical Distribution \& Logistics Management. Vol. 44, n. ${ }^{\circ}$ 6, págs. 434-463.

HUO, B.; HAN, Z.; CHEN, H.; ZHAO, X. (2015). «The effect of high-involvement human resource management practices on supply chain integration». International Journal of Physical Distribution \& Logistics Management. Vol. 45, n. ${ }^{\circ} 8$, págs. 716-746.

HUO, B.; YE, Y.; ZHAO, X.; SHOU, Y. (2016). «The impact of human capital on supply chain integration and competitive performance». International Journal of Production Economics. N. ${ }^{\circ}$ 178, págs. 132-143.

JIN, Y.; HOPKINS, M. M.; WITTMER, J. L. (2010). «Linking human capital to competitive advantages: Flexibility in a manufacturing firm's supply chain». Human Resource Management. Vol. 49, n. ${ }^{\circ}$ 5, págs. 939-963.

JORDAN, C.; JORDAN, C.; BAK, O.; BAK, O. (2016). «The growing scale and scope of the supply chain: a reflection on supply chain graduate skills». Supply Chain Management: An International Journal. Vol. 21, n. ${ }^{\circ}$ 5, págs. 610-626.

\section{Milena Gómez Gedeño Milena.gomez@utp.ac.pa Profesora de Ingeniería Industrial. Universidad Tecnológica de Panamá}

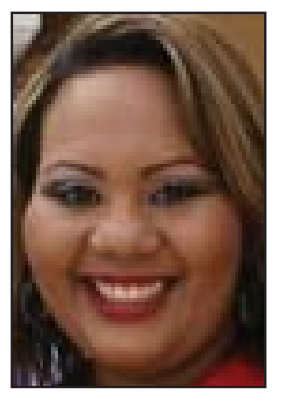

Doctora en Empresa por la Universidad de Barcelona (UB), obtuvo el Premio Extraordinario de Doctorado UB2015/2016 por su tesis «Incidencia de los recursos humanos en la gestión de la cadena de suministro». Además de ingeniera industrial, posee un máster en Supply Chain and Logistics (EAE-Universidad Politécnica de Cataluña, Barcelona), el máster de Investigación en Empresa (Universidad de Barcelona), así como un posgrado en Recursos Humanos (USMA). Actualmente trabaja como profesora-investigadora en la Universidad Tecnológica de Panamá (UTP) y es miembro del Sistema Nacional de Investigación de Panamá (SNI). 


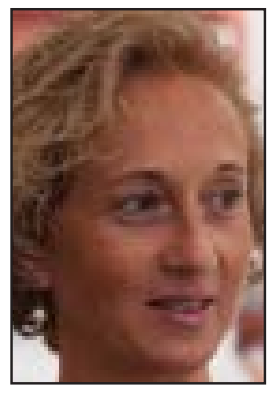

\section{Laura Guitart i Tarrés} laura.guitart@ub.edu

Profesora de Economía y Empresa (UB). Profesora colaboradora de los Estudios de Economía y Empresa (UOC)

Doctora en Ciencias Económicas y Empresariales por la Universidad de Barcelona e ingeniera industrial por la Universidad Politécnica de Cataluña. Es profesora del Departamento de Empresa de la Universidad de Barcelona desde 1994. Como investigadora ha publicado en diversas revistas académicas de prestigio internacional (como Industrial Management and Data Systems, International Journal of Market Research, Technology, Analysis \& Strategic Management o Innovation: Management Policy \& Practice) y ha participado en diversos proyectos competitivos (convocados y financiados por el Ministerio de Ciencia y Tecnología, por la Fundación Española para la Ciencia y la Tecnología (FECYT) o por la Generalitat de Cataluña). Es la investigadora principal del Grupo de Innovación Docente G.IDEA (http://www.ub.edu/gidea/), reconocido por la Universidad de Barcelona.

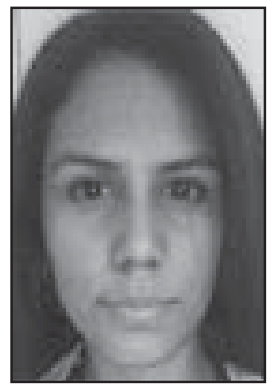

\section{Shantall Morantes Guerra} shantall.morantes@utp.ac.pa Universidad Tecnológica de Panamá

Estudiante de Licenciatura en Gestión de la producción Industrial en la Universidad Tecnológica de Panamá. Actualmente está realizando su tesis de grado «Modelo teórico para la medición del impacto de los recursos humanos en la cadena de suministro: caso panamá». Además, estudia Ingeniería industrial en la Universidad Latina de Panamá, en su octavo cuatrimestre.

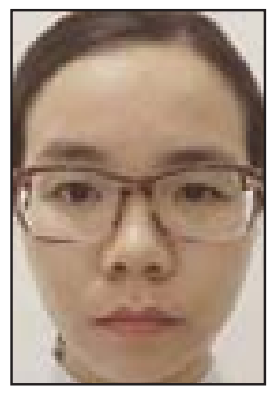

\section{Yohana Li Zeng} yohana.li@utp.ac.pa Universidad Tecnológica de Panamá

Estudiante de licenciatura en Gestión de la Producción Industrial en la Universidad Tecnológica de Panamá. Actualmente está realizando su tesis de grado, titulada «Modelo teórico para la medición del impacto de los recursos humanos en la cadena de suministro: caso Panamá». 
Los textos publicados en esta revista están sujetas -salvo que se indique el contrario- a una licencia de Reconocimiento 3.0 España de Creative Commons. Podéis copiarlos, distribuirlos, comunicarlos públicamente y hacer obras derivadas siempre que reconozcáis los créditos de las obras (autoría, nombre de la revista, institución editora) de la manera especificada por los autores o por la revista. La licencia completa se puede consultar en http://creativecommons.org/licenses/by/3.0/es/deed.ca.

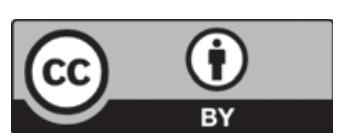

